\title{
Juliette: A model of sexual consent
}

\author{
Chandra Kavanagh
}

\begin{abstract}
The 'yes means yes' model of sexual consent and the political and ethical commitments that underpin this model have three fundamental disadvantages. This position unfairly polices the sexual expression of participants; it demands an unreasonably high standard for defining sexual interaction as consensual; and by denying the body's capacity for expressing sexual consent this model allows perpetrators of sexual violence to define consent. I argue that a critical examination of Marquis de Sade's novel Juliette can provide the basis for a model of sexual consent that avoids these problems by refraining from pre-judging the means by which consent is communicated.
\end{abstract}

Keywords: Sexual ethics, sexual consent, feminist theory, yes means yes, no means no, Marquis de Sade, embodiment

The slogan 'yes means yes' (Kearney 2015) has been adopted by many college antirape campaigns to replace the outdated slogan 'no means no.' (New 2014) 'No means no' and the political and ethical commitments that underpin this slogan have been rightly lambasted for demanding an unreasonably high standard to categorize a sexual assault as such, for policing women's sexual expression, and for disingenuously ignoring the expressive capacity of the body. In this paper I argue that the 'yes means yes' slogan, and the political and ethical commitments that underpin this slogan, retain variations of each of these three problems. The position represented by the 'yes means yes' slogan depends on the assumption that a verbal 'yes' is the ideal expression of consent and that other expressions of consent, such as bodily expressions, are less than ideal or even illegitimate. First, I argue that any theory of sexual consent dependent on such a hierarchy unfairly polices the sexual expression of participants. This is especially true for women, who have been conditioned in various ways to be unable or unwilling to express affirmative verbal consent. Second, I argue that a 'yes means yes' model is problematic insofar as it demands an unreasonably high standard for defining sexual interaction as consensual. Specifically, a theory of consent that privileges verbal consent over bodily consent requires defining any sexual experiences that begin with a verbal 'no', and is followed by a bodily expression of consent, as non-consensual. This definition obtains even in opposition to the judgment of the participants involved. Finally, by denying the body's capacity for expressing sexual consent and refusal, we allow perpetrators of sexual violence to define any conversation concerning sexual consent by focusing on the expressive capacities or choices of the survivors of sexual violation rather than focusing on 
whether or not consent is shared. In the last section of this paper I argue that a critical examination of the Marquis de Sade's novel Juliette can provide the basis for a model of sexual consent that does not pre-judge the means by which sexual consent is communicated. The notion found in Juliette that consent is active cooperation in the realization of shared projects provides the groundwork for a contextual account of consent capable of acknowledging the expressive capacity of the body. This acknowledgement makes it possible to validate the kinds of consenting practices often selected by women. Furthermore, a theory of consent that does not rely on a hierarchy that privileges verbal consent is able to investigate the experiences of partners who claim to have engaged in consensual sex despite token resistance, rather than redefining those experiences as non-consensual in opposition to the judgment of the participants involved. Finally, a notion of consent based on the Juliette model shifts the question of sexual consent from a focus on the capacity of survivors of sexual violence to express themselves, to the responsibility of violators to ensure that they have properly understood whether or not consent is shared.

\section{A Note Concerning Language}

Throughout this project I will be referring to victims and survivors of sexual violence with a female pronoun and perpetrators of violence with a male pronoun. This practice is widely used throughout the literature to acknowledge the gendered nature of sexual violence. (Little 2005, p.1325) It is also worth noting that sexual consent negotiations are almost always oversimplified in the literature in terms of a straightforward active/passive dynamic where a participant seeks consent from a single partner and that partner either consents or not. (Eskridge 1995, p. 49) While this type of straightforward negotiation is one way of understanding sexual consent that is taken up by this project, I contend that taking into account the contributions of the Juliette model to sexual consent will make space for a wide variety of sexual consent negotiations.

\section{Sexual Consent: An Overview}

Melaine Beres notes in her analysis of sexual consent literature that sexual consent is an undertheorized and disputed concept. She observes that much of the literature concerning sexual consent takes for granted a shared understanding of what it is to consent. (2007, p. 94)) In light of this gap in the literature, I will offer here a preparatory account of sexual consent before indicating the different ways in which this basic notion of sexual consent is developed in a 'yes means yes' type account and how it might be developed by taking into consideration consent as it is presented in Sade's Juliette. It is generally agreed upon that "sexual consent represents some form of agreement to engage in sexual activity." (Beres 2007, p. 97) However, two 
central questions at the heart of sexual consent remain contested. The first concerns the conditions of agreement or disagreement. The second concerns whether and in what ways sexual consent is psychological, behavioural or both.

One school of thought concerning the conditions of sexual consent, sometimes referred to as the "any yes" (Beres 2007, p. 98) position, claims either explicitly or implicitly that a yes, even under coercive circumstances, counts as consent. On the other end of the ideological spectrum is Catherine MacKinnon's much criticized position that in a patriarchal society women are in a coerced and oppressed position such that it is difficult to distinguish consensual from non-consensual sex. (1987, p. 88) This project takes up the moderate view, shared by other researchers such as Hall (1998) and Hickman and Muehlenhard, (1999) that consent requires the voluntary, or free approval of some person insofar as they are not being directly coerced. While more radical definitions may prove useful, this understanding captures our moral intuitions about sexual consent, for instance that a person cannot consent to sex while being threatened with violence, while also taking seriously sexual experience as it is described by oppressed people, for instance when a woman describes sex with her husband as consensual despite living in a male-dominated society.

So far the definition of sexual consent taken up by this project is that sexual consent is the free agreement to engage in some sexual activity. However, the form said agreement takes is heavily debated and therefore must be made explicit. Throughout the literature consent is sometimes referred to as a psychological state, sometimes it is referred to as a behavioural act, and often it is treated as a mixture of the two. (Beres 2007, p. 100-1) Again this project will take the moderate approach of understanding sexual consent both in terms of a psychological state of agreement concerning the sexual act that is being taken up, and in terms of behaviours that communicate consent. Beres argues "recognizing that the physical behaviours and cues that may take place during sexual activity are not consent in and of themselves, but may reflect the inner intentions of the individual" makes it possible to "disentangle the complexities of consent." (2007, p. 101) Considering both the psychological and behavioural aspects of consent makes it possible to investigate the complex interrelations between self and other that characterize consent. Hickman and Muehlenhard articulate the importance of both aspects of sexual consent when they claim that sexual consent is "the communication of a feeling of willingness." $(1999$, p. 259) Thus, this project understands sexual consent to be the free judgment to agree to engage in some sexual activity as communicated between those who are, or will be, engaged in the activity. This basic definition of sexual consent is developed in one direction by the position exemplified by the slogan 'yes mean yes' and in a slightly different direction by the notion of consent that comes out of Sade's Juliette.

\section{Sexual Consent: The 'Yes Means Yes' Position}

The understanding of sexual consent represented by the 'yes means yes' slogan is founded on the notion that a verbal yes is the ideal expression of consent. In her paper "Read Her Lips: An argument for a verbal consent standard in rape," LA Remick 
argues in favour of a theory of sexual consent "based on a norm of affirmative verbal consent. Under this standard 'no' would mean 'no,' 'yes' would mean 'yes,' and the lack of any verbal communication as to consent would be presumed to mean "no."' (1993, p.1105) Remick claims a standard mandating that the only recognized "signals of consent are verbal statements" (1993, p. 1121) can be justified insofar as it would protect women from becoming victims of non-malicious rape (that is, the unjust inference of compliance based on a woman's actions or inaction.) Remick maintains that a verbal 'yes' or 'no' is significantly less ambiguous than other kinds of expression. From this premise she argues that the requirement of a verbal 'yes' or 'no' leaves less space for misunderstanding than bodily expressions, when negotiating sexual consent.

Remick makes some space for bodily consent when she claims, "only overt behavior should be construed as consent." (2007, p. 1120) However, she collapses this space with the claim that the ambiguity of bodily consent allows for consent to be unjustly inferred, followed by the claim that "The problem of unjust inferences from a woman's actions or inaction could be resolved by a standard mandating that the only legally recognizable signals of consent are verbal statements." (Remick 2007, p. 1121) Mandating verbal consent as the only kind of legally recognizable consent clearly indicates verbal consent's position as the ideal kind of sexual consent while relegating bodily consent to a secondary, ambiguous or even illegal kind of consent.

In his paper defending an affirmative consent standard Nicholas Little argues that requiring sexual participants to request consent and respond affirmatively is an improvement on the traditional system. He reasons, "in cases of uncertainty about an individual's desires, the rational course of behavior is to ask, and then to give words in the answer their normal meaning." $(2005$, p. 1352) His commonsense approach is founded on the notion that communication in sexual situations ought to play by the same set of rules as other conversations. He maintains the position that "in normal conversation, 'no' is indeed taken to mean 'no.' Similarly, silence is not taken as meaning consent in other fields." (Little 2005, p. 1352 ) From this premise he reasons that negotiations of sexual consent ought to take place in this way despite his admission that "it is true that an affirmative consent standard would likely cause significant change in present sexual behavior." (Little 2005, p. 1359) Here Little agrees with Remick that verbal consent is the ideal kind of consent, and that all sexually active people should be required to express their sexual consent in this way.

Beres' analysis indicates a trend in the literature wherein verbal consent is treated as the ideal consent. In addition to several other works she notes that Pineau's notion of communicative sexuality makes some space for bodily consent, but continues to privilege verbal consent. Pineau argues that sexual consent is communicative and "ideally this communication would be verbal, although it does not need to be." (Beres, 2007 p. 102) There are two ways in which we can understand verbal consent as ideal. The first is that verbal consent is ideal in so far as the legal process is concerned. The second way verbal consent could be understood as ideal sexual consent is in terms of a normative claim. In other words, we ought to be encouraging or compelling sexually active people to take up this manner of negotiating sexual consent. We can easily understand verbal consent as legally ideal, either because it is ideal that a verbal response was given for purposes of legal clarity, or that an affirmative 
verbal consent standard is an improvement on current consent laws and policies. I will remain agnostic concerning the understanding of consent that ought to be enshrined in law. On the other hand, in terms of understanding affirmative consent as a normative claim, I will argue that the 'yes means yes' position demands an unreasonably high standard to define sexual interaction as consensual. Furthermore, such an understanding of sexual consent unfairly polices the sexuality of partners from whom consent is sought, and this is especially true for women. Finally, privileging verbal consent allows perpetrators to determine the conversation concerning sexual consent by denying the expressive capacity of the body.

\section{Challenges to The 'Yes Means Yes' Position}

The first problem with the 'yes means yes' position, and the hierarchization of verbal over bodily consent, is that this understanding of sexual consent unjustly polices the expression of sexual consent. This is especially true for women, who have reported a tendency to choose bodily expressions of consent. (Beres 2007, p.104) Remick argues that "a new consent standard based upon affirmative verbal consent" would be a step toward ensuring the freedom for women to "engage in sex however they choose." (1993, 1107) However, it seems clear that requiring verbal consent quite obviously limits women from engaging in sex however they choose. Specifically, it prevents them from engaging in sex wherein they do not give verbal consent. Even when we consider the begrudging space left for bodily expression in this hierarchy of consent, the expression of the body is still treated as lesser consent and the ambiguity of bodily consent is played up. As a result, any bodily expression intended to indicate consent must be overt enough to overcome the assumption that consent ought to be verbal. Furthermore, a verbal 'no', despite obvious disingenuousness, cannot be trumped by bodily expression. The assumption that bodily consent is less authoritative than verbal consent along with verbal consent's position as ideal sexual consent limits participants to a kind of bodily consent that is almost as restrictive as affirmative verbal consent on this account.

When we consider this restrictive understanding of sexual consent, alongside acknowledging women's cultural and historical location as those whose sexuality has often been silenced (Holland et. al. 1994), restricting consensual sex to overt verbal or non-verbal consent results in the refusal to sanction the kind of sexual consent that women are most likely to use. Even if a critic wishes to deny they ways in which women are conditioned to be unable or unwilling to express their consent, research attests to the fact that women choose to consent in subtle physical ways. Hickman and Muehlenhard record the lived reality of how women consent to sex in their paper "By the Semi-Mystical Appearance of the Condom: How Young Women and Men Communicate Sexual Consent in Heterosexual Situations." They found that when women were asked how they typically consented to sex, physical consent was the most highly rated category followed by verbal consent. Indicators of physical consent were often as subtle as "a look" or "a smile." $(1999$, p. 258) These subtle methods of bodily consent are unlikely to provide the affirmative consent required 
by the 'yes means yes' model given that any kind of bodily consent, even overt bodily consent, is less than ideal. Furthermore, such methods of expressing consent could certainly not overcome a token refusal. Thus, an approach that prioritizes verbal consent over the expressive capacity of the body at best ignores the manner in which many women and passive partners choose to consent, and at worst outlaws the limited methods of sexual consent most comfortable for women.

Given the practice of subtle bodily consent that is often employed by women, a 'yes means yes' model that privileges of verbal expression above bodily expression results in redesignating consensual sexual experiences as non-consensual in opposition to the judgment of the participants. There are countless examples of sexual relations that would not be classified as consensual under the parameters dictated by a 'yes mean yes' approach, but that the participants would classify as consensual. Kitzinger and Frith note:

The wide- spread use of 'token resistance' (saying 'no' but meaning 'yes') has been welldocumented and studies have repeatedly found that about 40 percent of US female undergraduates report saying to their dates that they did not want to have sex when actually they 'had every intention to' and were 'willing to engage in sexual intercourse' (Muehlenhard and Hollabaugh, 1988; Muehlenhard and McCoy, 1991; Sprecher et al., 1994). (1999 p. 306)

Under a 'yes means yes' model the sexual interaction that these women engaged in could not be consensual. In a hierarchy that privileges speech over bodily expression, no bodily behavior that these women engaged in could negate the privileged verbal refusal. Yet, these women describe themselves as willing, and having every intention to engage in the anticipated shared projects. Such a redefinition of a widespread, shared experience is difficult to justify. It is typical of modern, western society to trust people to judge whether or not they consented to sex. For instance in Canada, "in the vast majority of sexual assault cases, it is assumed that an adult complainant is capable of giving consent to sexual activity. Unless there is evidence of intoxication, impaired consciousness, or mental disability, capacity is rarely mentioned." (Benedet \& Grant 2007, p. 269) In other words, treating someone as incapable of consenting, or questioning their ability to judge themselves as consenting requires a clear justification such as profound mental incapacity or impairment. It is outrageous to classify 40 percent of undergraduate women as incapable of judging whether or not they consented to a sexual activity in order to honour the privileging of verbal consent over bodily consent that a 'yes means yes' approach to consent demands.

So far I have argued that the "yes means yes" model unjustly polices sexual expression, especially the sexual expression of women. Furthermore, this approach classifies a wide variety of sexual relations as non-consensual when the people engaged in the experience would classify those same activities as consensual. Finally I will argue that the 'yes means yes' model, and the privileging of verbal expression over bodily expression that underpins this model, denies the expressive capacity of the body, thus allowing perpetrators to set the parameters of a discussion of sexual consent by assuming that bodily expression is more ambiguous than verbal expression. I will provide three reasons to believe that the ambiguity of bodily consent has been exaggerated. First, there is a great deal of evidence that people have a sophisticated ability to read one another's bodily cues. Second, miscommunication theory puts the onus on the victim's expressive capacity. Claiming that sexual consent or 
refusal has been miscommunicated has proven a useful tool for violators to disingenuously defend their actions, and playing up the ambiguity of bodily expression is central to this tactic. Finally, human conversational interaction can be just as ambiguous as bodily interaction, especially within a sexual context, as we have seen in our discussion of token refusal. Thus, the privileging of verbal over bodily expression unjustly denies the ways participants can and do express sexual consent and refusal with their bodies.

Kitzinger and Firth found that sexual refusals followed culturally normative patterns and "both men and women have a sophisticated ability to convey and to comprehend refusals, including refusals which do not include the word 'no." (1999, p. 295) Given this ability to discern between nuanced acceptances and rejections it seems implausible that individuals who are able to understand one another well outside of a sexual context would lose that capacity within a context where sexual consent is being negotiated. Conversation analyst Michael Moerman claims "in any society, the recurrent and systematic attainment of misunderstanding between members of social categories who regularly converse with one another must thus be regarded as an artful, complicit, and damning accomplishment." (1988, p. 45) In other words, there can never be a clear enough kind of expression to reach a person that chooses not to understand. Beres' investigation into sexual communication revealed that many research participants "have shared rich descriptions of communications that take place through breathing, closeness of partners, eye contact and other subtle behavioural cues." (2007, p. 106) She notes that these people demonstrate a deep awareness of the nuances that indicate consent and refusal. Furthermore she argues that the conventional, but under theorized, practice of expressing consent and refusal through the body suggests "that it is possible to untangle some of the subtle ways that consent is communicated and reinforces [her] conviction regarding the importance" (Beres 2007, p.106) of researching bodily expressions of consent and refusal. The sophisticated ability people share to determine consent and refusal both outside and within a sexual context has been well documented. That the ambiguity of bodily consent is rarely at issue in other contexts, but consistently reiterated in a sexual context, suggests that there may be reasons aside from seeking a thorough understanding of sexual consent that motivate highlighting the ambiguity of bodily consent.

The supposed ambiguity of sexual consent and refusal as the body expresses it has historically provided an excuse to blame sexual violations on the supposed inability of survivors (especially women) to express themselves. Miscommunication theory refers to the notion that survivors of sexual violence were unable to express their dissent adequately, and thus the perpetrator did not know that his actions were unwanted. It has been demonstrated that "the miscommunication model of date rape is a useful resource for defendants in sexual assault tribunals seeking to construct themselves as innocent: complainants are represented as deficient in their efforts to signal non-consent." (Kitzinger \& Firth 1999, p. 295) In this circumstance the onus is on the survivor to prove that she did enough to inform the violator that his advances were unwanted. Similarly, a 'yes means yes' model that continues to define bodily consent as inferior to verbal consent retains the mirror image of the problem that arises when we make survivors responsible for 'adequately' expressing refusal. The slogan 'yes means yes' allows the perpetrators of violence to define the conversation 
of sexual consent by focusing on the survivor's ability to express herself adequately rather than the responsibility of the perpetrator to make sure his understanding of his partner's consent is clear. The central claim of Kitzinger and Frith's project is that "the problem of sexual coercion cannot be fixed by changing the way women talk." (1999, p. 311) This applies equally to both consent and refusal. It is difficult to defend taking seriously the capacity of the body to express refusal while denying the body's capacity to express agreement.

In addition to the use of miscommunication theory to defend sexual violations, and the documented capacity for members of social categories to interpret one another's subtle or complex acceptances and refusals, verbal communication can be just as ambiguous as bodily expression especially in a sexual context. On the whole, "human conversational_interaction is indeed intricately complex: 'yes' may some- times mean 'no', 'no' may sometimes mean 'yes', and the word 'no' is not necessarily part of a refusal." (Kitzinger \& Firth 1999, p. 310) Theorists such as Little make the claim that in sexual situations, as in other situations, "the rational course of behavior is to ask, and then to give words in the answer their normal meaning." (2005, p. 1352) However, as conversational analysts like Kitzinger and Firth or Hickman and Muehlenhard note, verbal indications of consent like 'yes' and 'no' are often employed in unexpected ways that nonetheless communicate and are understood as expressing consent and refusal, both within sexual contexts and without. In her analysis of the literature Beres notes that in all four reported studies concerning sexual consent, "non-verbal behaviours are used more frequently than verbal behaviours to communicate consent." (2007, p.104) While I, along with other many other theorists, (Kitzinger \& Firth 1999 p. 297) argue that women may be socialized such that it is difficult to verbally express consent, it could also be the case that people frequently choose bodily expressions of consent because bodily expressions are equally clear, less ambiguous than, verbal expression within a sexual context.

The 'yes means yes' approach to sexual consent retains variations of problems found in a traditional 'no means no' approach. A hierarchy that privileges verbal consent over bodily consent unfairly polices the means by which consent is expressed. Such an approach demands the redefinition of consensual sexual experiences as non-consensual in opposition to the perceptions of the participants involved. And finally, assuming the ambiguity of bodily consent allows perpetrators of violence to define sexual consent in terms of the survivor's capacity for expression, rather than focusing on the action required to understand consent. It is my contention that Sade's novel Juliette can provide insight into what a theory of consent that does not prioritize verbal expression over bodily expression might look like. Sexual consent as it is depicted in this novel suggests a way of understanding consent that avoids the three challenges raised by the 'yes mean yes' approach. My aim is not to give an exhaustive account of the theoretical apparatus that a study of Juliette's behavior could provide to a robust theory of bodily consent. Instead I seek to begin such an account, and prove it has the potential to provide valuable contributions to a theory of sexual consent that rejects a hierarchy of verbal over bodily expression. 


\section{Sexual Consent: The Juliette Model}

The Juliette model of consent begins by admitting the power that the body has to express consent as opposed to beginning with a focus on the ambiguity of bodily expression. In a discussion that can be applied to better understand bodily expressions of sexual consent, one of Juliette's initial tutors Madame Delbene propounds what she takes to be a foundational truth of nature. She says, "let mental feelings produce movements in living bodies, let the movement of bodies excite sentiments in the soul." (Sade 1968, p. 40) This chain of events, beginning with feeling expressed in the body, which goes on to further excite the soul provides a description useful for unpacking the process of bodily consent. It is though the sanctioning of the movements of the body that consent can both be made recognizable, and contribute to the project to which one is consenting. The addition of affirmative verbal consent here is not prohibited, but nor is it required in order to make consent intelligible.

The apparatus for this theory of consent is further developed by Juliette's lover Norceuil's contribution to what it means to consent when he informs Juliette, "So long as the schemes [your intellect] invents sort well with mine I shall always consent to them, better still, actively cooperate in their realization." (Sade 1968, p. 202) Norceuil's claim that the ideal consent is active cooperation in the realization of shared projects is capable of replacing affirmative verbal consent as the ideal kind of consent. This construction of consent allows for a multiplicity of methods for expressing consent and non-consent rather than depending on a hierarchy that privileges verbal consent.

Sade's redefinition of sexual consent in terms of the active co-operation in shared projects avoids the three challenges raised by the 'yes means yes' approach. First, rather than policing the style in which people (especially women) choose to consent, the notion of active cooperation in shared projects makes way for a variety of overt and subtle verbal and physical ways of consenting. Second, this way of understanding sexual consent classifies consensual and non-consensual sex in a way that coheres more closely with participants' experiences of consenting to sexual interaction than the 'yes means yes' model. Finally, understanding consent in terms of active co-operation allows for the possibility that verbal and bodily expressions of consent may be more, less, or equally expressive. Admitting the importance of context and the ambiguity of all kinds of expression avoids a definition of consent that only interrogates the expressive capacity of those from whom consent is being sought, and attends to the responsibility that those who are seeking consent have to ensure they have properly understood what is being expressed.

Understanding sexual consent in terms of active participation in shared projects, rather than depending on a hierarchy that privileges verbal consent, avoids unjustly policing the expressive bodily style of sexual consent that people, especially women and passive partners, choose to use. As a result of a definition of sexual consent that withholds judgment concerning the manner in which consent is expressed, bodily expressions of consent and refusal can be considered equal to verbal consent in legitimacy. Women who choose to consent with a "look or a smile" (Hickman \& Muehlenhard 1999, p. 258) have consented in a way that is just as meaningful as 
when women choose to consent with an affirmative verbal response. This position acknowledges the reality that people express consent and refusal in a wide variety of ways that all deserve to be taken seriously.

Another result of reserving judgment concerning the manner in which sexual consent is expressed, and attending to an understanding of sexual consent that takes into account consent as active participation in shared projects, is that space is created for participants to give voice to their own experiences in terms of consent and refusal. Rather than telling 40 percent of undergraduate women that they were wrong when they experienced engaging in consensual sex despite saying no, it is possible to interrogate those experiences in order to provide a better understanding of what sexual consent is like. Rather than introducing a consent standard that demands "significant change in present sexual behavior" (Little 2005, p. 1359), under the Juliette model of consent the possibility is open to explore how sexual consent is already negotiated and heed the call (Kitzinger \& Firth 1999, p. 311) for further theoretical development concerning bodily consent.

Finally, an understanding of sexual consent that defines sexual consent in terms of active participation of any kind, rather than privileging verbal consent, also avoids the pitfalls that come with denying the expressive capacity of the body. Instead of treating bodily consent as less ideal than verbal consent, considering sexual consent in terms of active cooperation in shared projects allows space to refocus from the expressive choices of survivors of sexual violence to the responsibilities of all participants to ensure sexual engagement is consensual. In light of the legitimation of all kinds of consent and refusal from subtle physical gestures, to clear verbal statements, to meaningful silence, alongside the acknowledgement that all expressions of consent can be clear and ambiguous, verbal and bodily expressions are assumed to meaningfully convey consent or refusal. Thus, the onus is on the perpetrator of sexual violence to provide an account of his actions for determining and respecting sexual consent.

One of the central justifications for the position that underlies the "yes means yes' slogan is that other theories of consent define the partner from whom consent is being sought, usually the woman, as always, already consenting to sex. Proponents of affirmative consent like Remick and Little claim "the traditional system works on the assumption that consent can be presumed unless withdrawn by the women." (Little 2005, p. 1347) Little argues that a standard of affirmative consent is justified insofar as it protects women from the dangers presented by the misuse of miscommunication theory. If it is the case that a 'yes means yes' model disrupts the presumption of consent while the Juliette model retains it, then there may be a good reason to select a 'yes mean yes' model over the Juliette model. However, while a 'yes means yes' model avoids the problem of presumed consent, a definition of sexual consent that does not prioritize verbal or bodily consent also avoids this pitfall.

When we understand sexual consent in terms of active cooperation in the realization of a given project, simple silence or a lack of resistance alone is not enough to claim that a person has given her consent. Rather, attitude, context, history and subtle cues must all be considered when interpreting what a given silence is meant to convey. For instance, in the case of the Burlington tragedy Sade's character Borchamps murders the husband, sister and father of his innocent love interest Clotilda. 
These were acts to which Clotilda had "consented only through silence." (Sade 1968 p. 841) As a result of Clotilda's silent consent, coupled with her lack of "active cooperation" (Sade 1968, p. 202) in the realization of the project it is Borchamps' claim that she is not at all responsible for the acts. He explains, "Clotilda cannot be considered even partly responsible for any of the pieces of mischief that I have just related to you. The passive instrument of my maneuvers, she was in no wise their cause." (Sade 1968 p. 840) In this case her silence indicates that she has not consented, while in other places in the work silence does indicate consent. For instance, this is the case when Juliette claims that her partners so desire her that she is "able to read consent in their expressions." (Sade 1968 p. 353). When this notion of silence as both expressive and ambiguous is applied to sexual consent it ameliorates any concern that the Juliette model of consent puts women in the position of always, already consenting. It also avoids putting women in the position of always, already refusing. Rather it reflects the reality of the silent person, namely that without more information we cannot know what her silence means.

The communication between individuals that allows for both to willingly participate in shared projects is complex. We say 'no' when we mean 'yes' and 'yes' when we mean 'no'; we consent and refuse with eye contact, facial expressions, touch and reactions to touch. Positions like 'yes means yes' try to simplify and hierarchize sexual consent in an effort to clarify which sexual interactions can be classified as consensual and which can be classified as not consensual. However rather than investigating the ways in which people actually negotiate consent, the 'yes means yes' model prescribes one style of expressing consent, verbal consent, and relegates bodily consent to a subordinate position. As I have shown, such hierarchization is a problem because it unjustly polices sexual expression, especially the types of sexual expression chosen by women. The 'yes means yes' position demands the redefinition of many sexual experiences as non-consensual, in opposition to the judgment of the participants involved. And finally this position is a problem because it allows perpetrators of sexual violence to set the parameters of sexual consent by ignoring the expressive capacity of the body. As Kitzinger and Firth write, "“yes means yes, and no means no' may make a good campaign slogan, but it is neither a description of actual human behaviour, nor a suitable prescription for dealing with the sexual coercion." (1999, p. 311) I have argued that an understanding of sexual consent based on the notion found in Sade's Juliette, namely that consent is active co-operation in the realization of shared projects, avoids these three problems by doing away with the hierarchy of verbal consent over bodily consent.

\section{References}

Benedet, J. \& Grant, I., 2007. Hearing the Sexual Assault Complaints of Women with Mental Disabilities: Consent, Capacity and Mistaken Belief. McGill Law Journal. 52(1). 243-289.

Beres, M.A., 2007. 'Spontaneous' Sexual Consent: An Analysis of the Sexual Consent Literature. Feminism \& Psychology. 17(1). 93-108. 
Eskridge, W.N., 1995. The Many Faces of Sexual Consent. William \& Mary Law Review, 37(1), 47-67.

Hall, D., 1998. Consent for Sexual Behaviour in a College Student Population. Electronic Journal of Human Sexuality [Online], 1(1). Available from: http://www. ejhs.org/tocv1.htm [Accessed June 2015].

Hickman, S.E. \& Muehlenhard, C.L., 1999. "By The Semi-mystical Appearance Of A Condom": How Young Women And Men Communicate Sexual Consent In Heterosexual Situations. Journal of Sex Research, 36(3), 258-272.

Holland, J. et al., 1994. Power and Desire: The Embodiment of Female Sexuality. Feminist Review, 1(46), 21-38.

Kearney, L., 2015. All NY colleges to adopt 'yes means yes' sex assault policy. Reuters.

Kitzinger, C. \& Firth, H., 1999. Just Say No? The Use of Conversation Analysis in Developing a Feminist Perspective on Sexual Refusal. Discourse and Society. 10(3), 293-316.

Little, N. J., 2005. From No Means No to Only Yes Means Yes: The Rational Results of an Affirmative Consent Standard in Rape Law. Vanderbilt Law Review. 58(4). $321-363$.

MacKinnon, C.A., 1987. Feminism unmodified: discourses on life and law, Cambridge, Mass.: Harvard University Press.

Moerman, M., 1988. Talking culture: ethnography and conversation analysis, Philadelphia: University of Pennsylvania Press.

New, J., 2014. More college campuses swap 'No means no' for 'Yes means yes.' PBS.

Remick, L.A., 1993. Read Her Lips: An Argument for a Verbal Consent Standard in Rape. University of Pennsylvania Law Review 141(3), 1103-151.

Sade, M.de, 1968. Juliette, New York: Grove Press, Inc. 